\title{
Deplacement Involontaire De Populations Et Conflits Fonciers A Ayaou-Sokpa (Sous-Prefecture D'ayaou- Sran, Centre De La Cote d'Ivoire)
}

\author{
Oura Kouadio Raphaël \\ Géographe, Maître de recherche \\ CRD, Université Alassane Ouattara, Côte d'Ivoire \\ Kouassi Kouamé Sylvestre \\ Géographe, Maître-Assistant \\ Université Alassane Ouattara, Côte d'Ivoire \\ Koffi Simplice Yao \\ Géographe, Maître assistant \\ Université Péléforo Gon Coulibaly, Côte d'Ivoire
}

\section{Résumé}

La construction du barrage de Kossou a nécessité au début des années 1970, le déplacement de six villages de leur site périphérique au fleuve Bandama vers Assamabo, un autre village de la même communauté ayaou, situé à une quinzaine de kilomètres. Pour atténuer les effets du déplacement, des habitats modernes et des équipements socio-collectifs ont été réalisés. En outre, des terres agricoles ont été négociées dans le village d'accueil. Mais, les terres s'étant raréfiées après plus de 40 ans, on assiste maintenant à des conflits de tout genre notamment entre les autochtones et leurs hôtes. Cette étude, qui repose sur des données d'enquêtes qualitative et quantitative auprès de divers acteurs d'Ayaou-Sokpa, fait l'analyse des conflits fonciers liés à la réinstallation des populations déplacées dans le cadre de la construction du barrage. Il en ressort que $24 \%$ des conflits évoqués par les enquêtés relèvent de ceux existant entre les différents villages. Le deuxième type de conflit, celui qui oppose les agriculteurs aux éleveurs, représente selon les avis recueillis, $48 \%$ des cas vécus dans le monde rural ayaou. Le dernier type, dans une proportion de $28 \%$, engage les agriculteurs eux-mêmes. Il ressort aussi que les personnes en sérieux désaccords recourent progressivement au mode de règlement moderne du fait de quelques signes de faiblesse dans la voie coutumière.

Mots-clés : Ayaou-Sran, Projet d'aménagement ; barrage de Kossou ; conflit foncier; gestion foncière 


\title{
Involuntary Displacement Of Populations And Land Conflicts In Ayaou-Sokpa (Subprefecture Of Ayaou- Sran, Centre Of Côte d'Ivoire)
}

\author{
Oura Kouadio Raphaël \\ Géographe, Maître de recherche \\ CRD, Université Alassane Ouattara, Côte d'Ivoire \\ Kouassi Kouamé Sylvestre \\ Géographe, Maître-Assistant \\ Université Alassane Ouattara, Côte d'Ivoire \\ Koffi Simplice Yao \\ Géographe, Maître assistant \\ Université Péléforo Gon Coulibaly, Côte d'Ivoire
}

\begin{abstract}
In the early 1970s, the construction of the Kossou dam required the relocation of six villages from their peripheral site near the Bandama River to Assamabo, another village of the same Ayaou community located some fifteen kilometres away. To mitigate the effects of the displacement, modern housing conditions and public facilities were built. In addition, agricultural plots of land were negotiated in the host village. However, faced with land scarcity after more than 40 years, we are now witnessing conflicts of all kinds, particularly between natives and their hosts. This study, which is based on both qualitative and quantitative survey data to various stakeholders in AyaouSran, analyzes land conflicts related to the resettlement of displaced populations as part of the construction of the dam. It appears that $24 \%$ of the conflicts mentioned by the respondents are among those existing between the different villages. The second type of conflict, which mainly occurs between farmers and pastoralists, are said to represent $48 \%$ of the cases experienced in the rural world. The last type, in a proportion of $28 \%$, involves the farmers themselves. It also appears that the belligerents are progressively resorting to the modern mode of settlement because of some signs of weakness in the customary way.
\end{abstract}

Keywords: Ayaou-Sran; planning project; Kossou dam; land conflict; land management 


\section{Introduction}

Après son accession à l'indépendance en 1960, l'Etat ivoirien dans sa politique de développement, a mis en place des projets d'envergure d'aménagement de son territoire. C'est dans cette optique que s'inscrit l'Autorité pour l'aménagement de la Vallée du Bandama (AVB).

Selon MEMPD ${ }^{1}$ (2006: 10), «l'AVB a été créé en juillet 1969 et couvre environ $40000 \mathrm{~km}^{2} \gg$. Dans la pratique, cette autorité avait pour mission d'élaborer une stratégie de développement régional suite à l'aménagement des eaux de la vallée du Bandama. C'est ainsi que le barrage de Kossou a été construit dans les années 1970. Mais, la construction de ce projet structurant pour toute la région voire tout le pays, a nécessité le déplacement de plusieurs communautés villageoises notamment les Ayaou. Plus de 75000 personnes décrétées sinistrées, dont les villages (au nombre de 100) devant être inondés, ont été contraintes à un déplacement de leur terroir traditionnel vers d'autres, moins soumis à la pression de l'eau (Ori, 1998 : 1). Souvent, les nouveaux venus se réinstallent dans des secteurs déjà occupés soit par des ethnies différentes ou soit par la même ethnie (Lassailly-Jacob, 1983 : 221). C'est ainsi que la communauté ayaou a été déplacée et relogée sur le terroir du village voisin Assamabo (carte $\left.n^{\circ} 1\right)$.

Carte 1 : Présentation de la zone d'étude

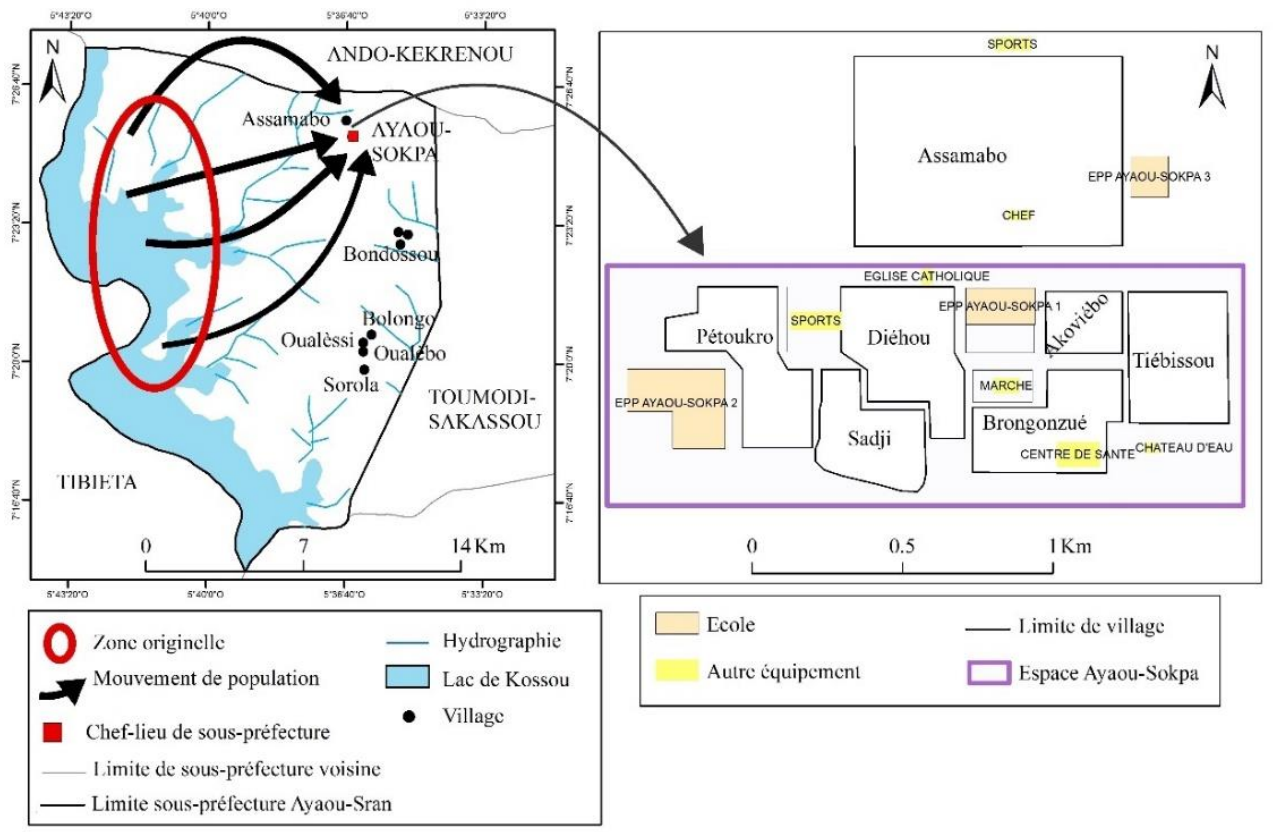

Source : BNETD-CCT, 2012 Oura K., 2017

${ }^{1}$ Ministère d'Etat Ministère du Plan et du Développement. 
Pour favoriser ce départ, le projet a mené des actions en faveur des populations affectées. Ainsi, des habitats modernes et des équipements sociocollectifs ont été réalisés pour ces populations. Toutefois, le projet de réinstallation, n'ayant pas été mené comme une initiative de développement, a entrainé une détérioration des conditions de vie des populations (Diop et $a l$., 2009 : 5). En effet, la question foncière, l'un des éléments fondamentaux de leur survie, a été sous-estimée dans ce programme de déplacement. Et sur les nouveaux espaces de vie, l'accession à la terre agricole pour ces nouvelles communautés est devenue difficile, puisqu'elles sont obligées de négocier de nouveaux espaces de culture.

Les populations déplacées n'ayant pas pour la plupart de terre pour pratiquer l'agriculture, leur principale activité, retournent sur leurs espaces de vie originels épargnés par l'extension de l'eau et restés à une quinzaine de kilomètres du nouveau village. Celles qui trouvent assez éloignés les anciens villages, continuent de quémander directement auprès du village d'accueil les compléments de terre nécessaires à leur subsistance (Lassailly-Jacob, 1983 : 224). Mais, aujourd'hui, les déplacés s'adonnent à l'anacardier, une culture pérenne et l'une des plus rentables dans la région. Le développement de cette plante sur la terre de leurs hôtes entraîne des tensions foncières. A cette situation, s'est ajouté un nouveau cas de conflit, celui qui oppose aujourd'hui les agriculteurs de retour aux éleveurs peuls. Ces derniers ont profité de la crise militaro-politique de 2002 pour s'installer massivement avec plusieurs troupeaux sur les terres abandonnées durant plus de trois décennies par la communauté ayaou. La sous-préfecture d'Ayaou-Sran est ainsi soumise à des conflits fonciers de tout genre dont les règlements deviennent de plus en plus difficiles.

Comment le déplacement de la communauté ayaou a-t-il été organisé dans le cadre du projet AVB ? Quelles sont les contraintes liées à la gestion foncière après la réinstallation de cette communauté ? Quelles sont les organisations locales pour faire face aux conflits induits par les contraintes foncières sur le nouvel espace d'accueil et les anciens terroirs ayaous ? Telles sont les questions auxquelles la présente recherche tentera d'apporter des éléments de réponse. L'objectif visé par cette étude est d'analyser les conflits fonciers liés aux contraintes foncières engendrées par la délocalisation des villages ayaous dans le cadre du projet AVB. La présente recherche part de l'hypothèse selon laquelle la faible prise en compte de la question foncière dans le projet $\mathrm{AVB}$ expose aujourd'hui les populations déplacées à des contraintes foncières qui sont source de conflits entre les différentes communautés. 


\section{Méthodologie}

L'approche méthodologique préconisée s'appuie sur un cadre théorique et conceptuel et un ensemble de techniques pour la collecte et le traitement des données.

\section{1- Cadre théorique et conceptuel}

Cette étude repose sur la théorie des conflits environnementaux qui traduit au mieux ces cas de conflits observés aujourd'hui sur les territoires ayaous. Du fait de la délocalisation de ces villages, on assiste en effet à la réduction des terres plus de quatre décennies après le projet d'aménagement du territoire de la vallée du Bandama.

\section{1- La théorie des conflits environnementaux}

Partant du fait que « la rareté engendre la violence », comme le dit Le Ster (2011 : 429), la présente recherche mobilise la théorie des "conflits environnementaux". En effet, les conflits liés aux ressources environnementales ont été analysés sous l'angle économique à travers le modèle établi par Thomas Homer-Dixon (1999). L'auteur décrit la façon dont le stress environnemental (la pénurie des ressources renouvelables provoquée par la dégradation environnementale, la croissance démographique et l'accès difficile aux ressources naturelles) déclenche nécessairement des conflits. La dimension démographique intéresse également la présente étude avec la forte pression humaine sur le nouvel espace d'accueil conséquente au regroupement de populations de plusieurs villages sur le terroir du seul village d'Assamabo. Ce déplacement ayant été fait depuis près de cinq décennies, le territoire ayaou connait naturellement un croît démographique alors que les ressources naturelles locales ne font que se restreindre dans un contexte où les pratiques agricoles n'ont pu se moderniser, comme le préconisait en partie le projet de l'Aménagement de la Vallée du Bandama avec l'introduction de nouvelles semences sur les petites exploitations de café créées. Cette recherche essaie donc d'établir le lien entre la raréfaction des ressources naturelles et les conflits en cours sur les espaces de culture des populations déplacées dans le cadre du vaste projet d'aménagement du territoire de la vallée du Bandama conçu par les autorités ivoiriennes.

\section{2- Le concept de l'Aménagement du territoire}

Les définitions du concept d'aménagement du territoire sont fonction des auteurs, soit qu'ils privilégient les finalités, soit qu'ils s'intéressent aux périmètres de compétence ou les modalités d'action conduites au nom de l'aménagement. Ainsi, selon Eugène Claudius-Petit, ancien Ministre Français de la Reconstruction et de l'Urbanisme, l'aménagement du territoire est « la recherche dans le cadre géographique, d'une meilleure répartition des 
hommes, en fonction des ressources naturelles et des activités économiques. Cette recherche n'est donc pas faite à des fins strictement économiques, mais bien davantage pour le bien-être et l'épanouissement de la population » (Communication en conseil des Ministres, février 1950 in Lacour et al., 2005 :7). Pour l'économiste Joseph Lajugie et al. (1979), « l'aménagement du territoire a pour fin, à la fois, de promouvoir la mise en valeur des ressources régionales et d'améliorer le cadre de vie et les conditions d'exercice des habitants, en atténuant les disparités régionales de développement économique et social par une organisation prospective de l'espace, reposant sur une orientation volontariste et concentrée des équipements et des activités ». La dimension prospective est essentielle en ce sens que les actes d'aménagement s'inscrivent dans la durée et vont donc conditionner l'avenir des populations concernées. A ce titre, ces actes méritent donc d'être pensés très tôt avec une prise en compte des futurs possibles et de leurs conséquences sur le territoire.

L'aménagement du territoire repose sur cinq principes fondamentaux et permanents qui guident toutes les initiatives en la matière. Il s'agit des principes de répartition ou de redistribution, de création, de réparation, de compensation et enfin, celui de protection. La prise en compte de ces principes d'action permet d'avoir « un bon aménagement » qui répond aux attentes du moment sans compromettre l'avenir. Il s'agira donc, dans cette contribution, d'analyser l'aménagement conduit dans la sous-préfecture d'Ayaou-Sran sous l'angle de son niveau de durabilité.

\section{2- Techniques de collecte et de traitement des données}

Les techniques du recueil de données reposent sur la documentation, l'observation sur le terrain, l'enquête par un questionnaire et par guides d'entretien. La revue documentaire a porté essentiellement sur les pratiques agricoles, la réinstallation de populations déplacées dans le cadre de la construction de barrages et sur les tensions foncières. Les articles de LassaillyJacob (1983) et de Ori (1984) ont été particulièrement utiles, pour leurs centres d'intérêts sur la question du déplacement des populations dans le cadre de la construction du barrage de Kossou. Quant à l'enquête de terrain, elle nous a permis de nous imprégner des réalités sociales du canton Ayaou-Sopka, notamment la gouvernance du foncier rural. Ce canton est constitué de six villages déplacés lors de la construction du barrage. L'enquête par entretien a été orientée d'une part, vers les autorités étatiques (agents agricoles et le souspréfet d'Ayaou-Sran) et d'autre part, vers les personnes ressources locales (le chef de canton, les chefs de village, le président du comité de gestion des conflits agriculteurs/éleveurs, les présidents des jeunes, les chefs de ménages ayaou et le chef des éleveurs). Quant au questionnaire, en l'absence d'une base de sondage, il a été adressé à 25 chefs de ménages dans chacun des villages de Pétoukro, Sadji, Brongonzué et Akoviébo. Ces trois derniers villages choisis 
ont la particularité d'être au cœur des conflits entre agriculteurs et éleveurs quand le choix de Pétoukro se justifie plutôt par l'occupation de son ancien terroir par Bondossou, un autre village de déplacés, de la communauté voisine sran.

Les données quantitatives ont été traitées à l'aide du logiciel Sphinx. Les données qualitatives ont quant à elles été retranscrites après collecte au dictaphone. Aussi, l'utilisation d'un GPS, pour la localisation du site des anciens villages et le logiciel ArcGIS ont été nécessaires pour la réalisation de la carte.

\section{Résultats et discussion}

1- L'AVB, un projet structurant dans la politique nationale d'aménagement du territoire avec des opportunités pour les populations locales ayaou

Les prévisions de la construction du barrage de Kossou annonçaient de nombreux dégâts matériels notamment l'inondation de nombreux villages périphériques au fleuve dont ceux de la communauté ayaou (Hauhouot, 2002 : 93). La mise en œuvre de ce projet a donc conduit au déplacement de ces populations. En effet, après l'accession du pays à l'indépendance en 1960, les gouvernants ont mis en place une politique hardie d'aménagement du territoire pour atteindre les objectifs de développement traduits dans les différents documents de planification dont les Perspectives décennales couvrant la période 1960-1970 (Hauhouot, 2002 : 93). Dans la partie centrale du pays, le grand rêve de l'aménagement du territoire était porté par le projet de l'Autorité pour l'Aménagement de la Vallée du Bandama (AVB) qui constituait également une chance d'amélioration des conditions de vie des populations.

\section{L'AVB, un projet phare de l'âge d'or de l'aménagement du territoire ivoirien}

Le déplacement de la communauté ayaou est intervenu au début des années 1970, lorsque l'Etat ivoirien a entrepris la construction du barrage de Kossou sur le fleuve Bandama. Autrefois, ce peuple vivait en effet dans de petits villages près du fleuve. Du fait de cette proximité géographique, le risque pour ces villages d'être submergés par l'eau était grand. Selon les autorités locales, les villageois avaient été avertis par les gouvernants de cette menace. C'est alors qu'il a été demandé aux populations de se déplacer vers un nouveau site, le terroir d'Assamabo, prévu pour leur réinstallation. Pour faciliter leur départ, le projet AVB a mené des actions en faveur des populations déplacées.

Le projet AVB constituait avec celui de l'Autorité pour l'Aménagement de la Région du Sud-ouest (ARSO), les deux grands exemples de régions-programmes initiés par l'Etat de Côte d'Ivoire durant les deux 
premières décennies de développement qualifiées d'âge d'or de l'aménagement du territoire ivoirien (Hauhouot, 2002: 181). Le projet s'étendait sur un territoire d'environ $40000 \mathrm{~km}^{2}$, soit environ $12 \%$ de l'espace national. Il couvrait la région administrative du centre-Bandama. Le contexte de la mise en place du projet est justifié par les nombreuses contraintes naturelles, humaines et économiques qui risquent d'entraver la marche de cette région dans la course au développement engagée par les autorités nationales (Hauhouot, ibid : 185). Devant ces constats, le projet AVB est pensé pour inverser la tendance d'appauvrissement continue qui se précisait dans cette région. Dans sa formulation, le projet avait deux grands espaces d'exécution qui étaient le Centre-Bandama et la région sud-ouest en zone forestière. Dans sa partie savanicole qui intéresse la présente recherche, l'objectif assigné à l'AVB, créé en juillet 1969, était d'aménager la région du projet par le développement de l'économie agricole. De façon spécifique, il s'agissait, entre autres, de lutter contre l'émigration massive de la main-d'œuvre issue de cette région, d'accroître le revenu des paysans pour réduire l'écart existant entre eux et ceux de la zone forestière, de favoriser l'apparition de véritables exploitations modernes associant agriculture et élevage, et enfin, de coordonner les relations agriculture-habitat rural par un aménagement cohérent de l'espace au profit des populations locales y compris celles qui ont été déplacées.

\section{2- L'AVB, une fenêtre d'opportunités pour les populations sinistrées ayaou}

Le déplacement de la communauté ayaou a été possible grâce aux actions menées par l'Etat à travers le projet AVB. En vue de rendre la réinstallation effective, chaque chef de famille a reçu un habitat de type moderne (une maison « en dur » et toit de tôle (photo $n^{\circ} 2$ ). Une école primaire, plusieurs puits équipés de pompes, une maison de jeunes, un hangar agricole et un terrain de sport furent également établis dans tous les nouveaux villages (Lassailly-Jacob, 1983 : 226). Au niveau d'Ayaou-Sran, de nouvelles routes ont-elles été ouvertes entre les nouveaux villages et celui d'Assamabo. En termes d'équipements, deux écoles primaires et un dispensaire ont été construits. En outre, de nouvelles variétés de café ont été octroyées aux populations déplacées pour faciliter la création de petites exploitations sur des terres négociées auprès des habitants d'Assamabo, un village de la même communauté ayaou. 


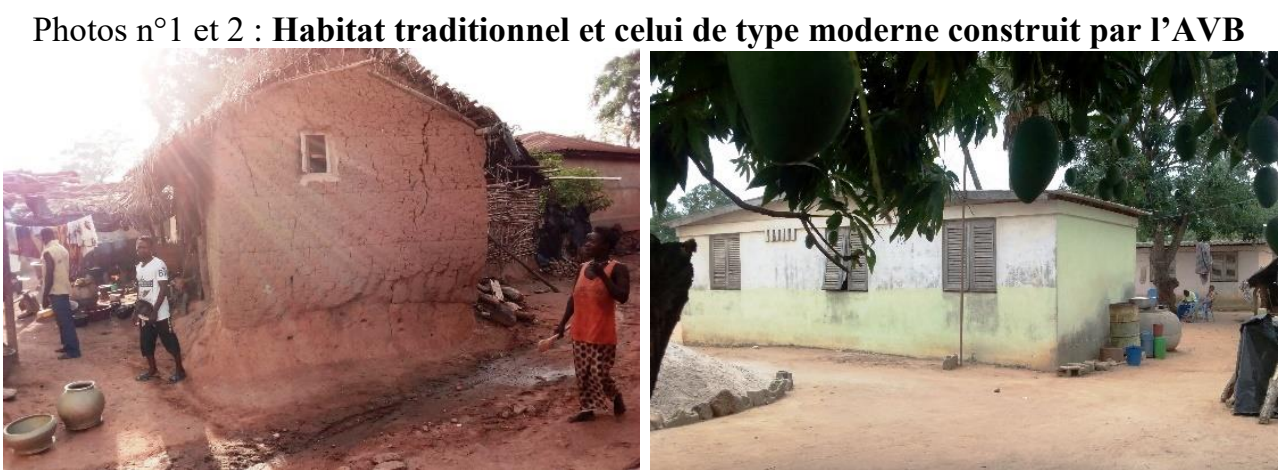

Source : Oura K., Janvier, 2017

Selon nos enquêtes, les habitants reconnaissent eux-mêmes que leur venue sur le nouveau site a été encouragée par la construction des habitations modernes. Outre l'installation de plusieurs équipements, le regroupement des six villages ${ }^{2}$ constitue pour eux, l'un des avantages du déplacement. En effet, la réunification de ces différents villages a encouragé la création de nouvelles infrastructures sanitaires (une infirmerie et un hôpital), scolaires (une école primaire et un collège de proximité) ainsi que l'électrification récente (en 1997) des villages. Elle a aussi facilité l'érection de leur nouvelle localité en sous-préfecture. Pour tous ces motifs, les paysans interrogés ne cachent pas leur satisfaction d'avoir été déplacés. Cependant, ce nouveau site d'accueil fait aujourd'hui face à des contraintes foncières qui sont à l'origine de nombreux conflits entre les populations.

\section{2- La faible prise en compte de la question foncière, un germe qui annonçait un avenir incertain du projet AVB}

Le déplacement de la communauté ayaou a rendu pénible la gestion des ressources foncières en raison de leur raréfaction sur le nouveau site. En effet, cette situation engendre aujourd'hui de nombreux conflits tant sur le nouveau site que sur les terres originelles que les populations déplacées tentent à nouveau de récupérer.

\section{1- La contrainte foncière, la conséquence sociale majeure du projet}

Pour la construction du barrage, le déplacement s'est fait sans compensation foncière pour les populations. Au niveau d'Ayaou-Sokpa, les six villages ont été réinstallés par le projet AVB sur le terroir du seul village d'Assamabo. Après plus de quatre décennies d'installation et de négociation des terres auprès du village d'accueil, Assamabo, la capacité de ces autochtones à céder encore des terres agricoles à leurs hôtes s'amenuise.

${ }^{2}$ Le canton Ayaou-Sokpa est constitué de six villages : Pétoukro, Diéhou, Sadji, Brongonzué, Akoviébo et Tiébissou. 
Face à ces contraintes foncières, on assiste à un retour des populations déplacées sur leurs anciens terroirs où ils disposent encore de quelques parcelles de terres pour pratiquer l'agriculture. Ce déplacement engendre toutefois une augmentation de la distance pour atteindre les champs. Des paysans parcourent jusqu'à 20 kilomètres par jour (l'aller et le retour). Les enquêtes ont révélé que $62,5 \%$ d'entre eux parcourent entre 10 et $20 \mathrm{~km}$ au quotidien. Les paysans connaissent donc dans l'ensemble des conditions de travail rendues difficiles par l'éloignement des terres agricoles.

L'éloignement des champs par rapport au site actuel réduit le dynamisme paysan. En effet, les exploitants d'âge avancé peinent à se rendre régulièrement au champ. Or, les enquêtes révèlent que la catégorie d'individus d'âge supérieur à 55 ans, reste importante dans la population agricole à AyaouSokpa ; ils représentent plus de $60 \%$ des personnes interrogées. A cet âge, il est déjà pénible de travailler dans les champs, quand la longue distance à parcourir constitue une contrainte supplémentaire pour ces derniers. Il s'ensuit une réduction de la taille des exploitations qui ne dépasse guère les 2 hectares. Le parcours quotidien de ces longues distances présente des risques sanitaires au regard de leur âge avec des conséquences systémiques à moyen et long termes sur la sécurité alimentaire et l'espérance de vie de ces derniers. En outre, les habitants doivent faire face aux difficultés de gestion foncière tant sur les anciens terroirs que sur le site actuel.

\section{2- Des conflits liés aux contraintes foncières}

La raréfaction des terres dans le village d'accueil et les derniers mouvements effectués par les populations pour faire face aux contraintes foncières ont engendré des conflits qui engagent une diversité d'acteurs. Les conflits sont de divers types allant de ceux opposant les paysans entre eux à ceux entre paysans et agriculteurs.

\subsection{1- Conflits inter-villages}

Les habitants d'Assamabo tolèrent de moins en moins la pression foncière induite par le regroupement de populations sur leur terroir. Ce qui justifie les cas de conflits entre ce village et les autres. En effet, $24 \%$ des conflits évoqués par les enquêtés relèvent de ceux existant entre les différents villages. Ce type de conflit intervient généralement quand les clauses du contrat de cession des terres aux déplacés pour le développement des cultures vivrières ne sont pas respectées. Ces tensions surviennent généralement lorsque certaines personnes jouissant d'un droit de gestion, tentent de le faire passer à un droit de propriété (Coulibaly, $2006: 5$; Ibo, $2012: 8$ ). En fait, avec l'avènement de l'anacardier, une culture hautement rentable, certains demandeurs de terres s'adonnent à la pratique de cette spéculation, sans 
solliciter l'avis des propriétaires terriens. Lorsque ces situations se présentent, ces derniers procèdent le plus souvent à l'arrachage des jeunes plants.

Le cas des tensions entre les villages de Bondossou et de Pétoukro témoigne du peu d'attention accordée par les décideurs à la question foncière. En effet, malgré qu'une bonne partie de son terroir ne soit sous la menace de l'eau lors de ce déplacement, Pétoukro s'est retrouvé auprès des autres villages ayaou, s'éloignant ainsi de ses terres. Si cette décision répondait à une volonté politique de regrouper les populations par communauté, le terroir abandonné par ce village a plutôt servi de site d'accueil à Bondossou, de la communauté voisine sran. Ainsi, face à la pression foncière actuelle, les habitants de Pétoukro conçoivent difficilement le fait que leurs terres originelles soient occupées par d'autres déplacés alors qu'ils n'en disposent pas eux-mêmes pour la pratique agricole. De plus, les paysans de Bondossou ont commencé à y développer l'anacardier, une culture arbustive qui sert de marqueur foncier. Un paysan de Pétoukro exprime ainsi son mécontentement :

«Les gens de Bondossou me demandaient la terre pour faire du vivrier. Aujourd'hui, à mon insu ils ont commencé à cultiver de l'anacarde sur ma terre. Lorsque j'ai découvert cela j'ai envoyé l'affaire chez le chef de canton, ensuite chez le sous-préfet. Malgré cela, ils continuent d'exploiter ma terre. »

Des conflits peuvent ainsi naître entre les paysans au fur et à mesure que s'accroissent les revendications sur la terre (PSDU, 2003 : 20). Des mésententes subsistent entre les habitants d'Assamabo et ceux d'Akoviébo. En effet, ces deux villages ont du mal à s'entendre sur les limites entre l'ancien site d'Akoviébo et celui d'Assamobo. Le témoignage d'un vieillard du village d'Akoviébo en est une illustration :

«Les gens d'Assamabo ont profité de notre longue période d'absence pour prendre nos terres de force. Aujourd'hui, on voit qu'ils ont largement dépassé leurs limites. Nous sommes allés plusieurs fois à la gendarmerie. Mais on n'a pas encore eu de suite favorable. »

Cette mésentente autour des limites des terroirs villageois débouche souvent sur des conflits, comme le révèle un autre paysan d'Akoviébo : «L'an passé, les gens d'Assamabo ont détruit les buttes d'un jeune d'ici sous prétexte que la terre sur laquelle il faisait ses buttes leur appartient ». Aussi, assiste-ton à des mésententes entre les habitants de Tiébissou, l'un des villages déplacés et ceux d'Assamabo. En effet, les deux villages revendiquent chacun l'espace qui abrite l'établissement secondaire public, nouvellement construit et dont l'Etat projette de dédommager les propriétaires terriens. 
Face à la récurrence des tensions entre les habitants d'Assamabo et leurs hôtes, le retour aux terroirs originels est vu par ceux-ci comme l'ultime solution à la crise foncière que leur déplacement a occasionnée. Mais, ce mouvement de retour révèle lui aussi de nouveaux cas de conflits sur les anciens sites qu'ils ont été contraints d'abandonner depuis bientôt cinquante ans.

\subsection{2- Conflits entre les paysans de même village}

Le deuxième type de conflit engage des agriculteurs au sein de la même communauté. Représentant une proportion de $28 \%$ des conflits cités dans la zone d'étude, ce type de conflit est lié aux difficultés qu'éprouvent les populations pour reconnaitre leurs terres épargnées par les eaux du barrage après plus de quatre décennies d'abandon. En effet, les paysans qui retournent maintenant sur ces terres ne peuvent reconnaître que de façon approximative les limites du patrimoine foncier de leur famille de départ. Le mode d'appropriation foncière se faisant de façon coutumière, la question de la délimitation constitue à n'en point douter la principale cause des tensions. A cela s'ajoute la volonté de certains acteurs à occuper de façon illicite ou de force de nouvelles terres. Ils profitent souvent de l'absence de certains propriétaires ou de leur invalidité pour étendre leurs exploitations au détriment de ces derniers. Ce propos recueilli d'un vieillard de 85 ans, du village de Sadji, traduit cet état de fait :

« Je n'arrive plus à marcher pour aller au champ. Un jour mon fils aîné est venu m'informer qu'un enfant de ce village est en train d'exploiter une partie de ma forêt. Lorsque je l'ai interpellé il me disait qu'il était en train d'exploiter la terre de son défunt père et que cette partie de la forêt ne m'appartient pas. Nous sommes allés à la cour royale de Sakassou plusieurs fois. Mais, la situation n'a pas encore changé. »

Ce propos témoigne des difficultés à définir clairement les limites des patrimoines fonciers. On assiste donc à des cas d'empiétements qui résultent de la mauvaise délimitation des terres (Koffié-Bipko et Adayé, 2015 ; Gaouli, 2008). Ainsi, le retour aux terres originelles ne garantit pas les populations déplacées d'un accès sécurisé aux terres. Au-delà de ces conflits intracommunautaires, il faut citer ceux entre les agriculteurs locaux et les éleveurs peuls.

\subsection{3- Conflits entre éleveurs et agriculteurs}

Ces dernières années, des conflits opposent aussi les agriculteurs revenus sur leurs terres aux éleveurs peuls sur les rives du lac. Ce type de 
conflit représente selon les avis recueillis, $48 \%$ des cas vécus dans le monde rural ayaou. Les terres étant restées vacantes, celles qui longent le fleuve ont été investies par des éleveurs ${ }^{3}$ qui en ont fait un lieu de pâturage de leurs troupeaux.

Cette arrivée d'éleveurs sur les rives du fleuve, dans le contexte de la crise militaro-politique de 2002, est maintenant source de conflits entre ces pasteurs et les paysans dont les exploitations se trouvent sur ces espaces pastoraux. Le plus souvent, les conflits agriculteurs/éleveurs surviennent lorsque les bœufs causent d'énormes dégâts lors du pâturage (Bernardet, 1986 : 33; Kohlhagen, 2002 : 14). Il s'agit notamment de la destruction de champs d'igname, de riz, de banane, de manioc, etc. Dans la plupart des cas, après de vaines tentatives de nier les faits qui lui sont reprochés, l'éleveur plaide pour une responsabilité involontaire en ce sens qu'il cherche à montrer que les dégâts commis relèvent d'un acte involontaire (Coulibaly, $2006: 7$ ). Ceux qui reconnaissent les faits dans ce cas, proposent une somme de 5000 FCFA $^{4}$ comme frais de dédommagement quels que soient les dégâts causés par leurs bœufs. Une somme que réfutent généralement les paysans. Cette situation entraîne très souvent la colère des agriculteurs qui tolèrent désormais très peu la présence de bœufs dans le voisinage de leurs exploitations, comme en atteste ce témoignage recueilli dans le village de Brongonzué :

«Un jour, les bœufs pâturaient dans mon champ. Lorsque mes enfants les ont vus, ils ont commencé à les chasser du champ. Sans demander à mes enfants, le Peul qui suivait les bœufs a commencé à frapper l'un d'eux avec son bâton qu'il prend pour suivre les bêtes. En se débattant, la machette qui se trouvait dans la main de mon fils a coupé le Peul. Il a aussitôt appelé les gendarmes. Ils sont venus me ligoter avec deux de mes fils pour nous envoyer à la gendarmerie. Après nous avoir interrogés, le commandant de brigade nous a demandé de revenir régler le problème à la sous-préfecture. Je n'ai pas encore oublié ce problème. J'ai gardé le bois du Peul que voici comme souvenir. »

Les agriculteurs étant pour la plupart éloignés de leurs champs, les conflits liés aux dégâts causés lors du pâturage sont récurrents de sorte qu'ils supportent de moins en moins la présence des troupeaux. Les cas de dégâts causés par les bœufs sont recensés dans le tableau 1 ci-après.

\footnotetext{
${ }^{3}$ Selon le Comité de Gestion, 21 éleveurs ont été dénombrés sur les rives du lac.

${ }^{4} 5000$ FCFA équivaut à environ 8 Euros
} 
Tableau $n^{\circ} 1$ : Récapitulatif des cas de dégâts enregistrés par le comité de gestion des conflits agriculteurs/éleveurs

\begin{tabular}{|l|l|l|l|l|l|l|l|l|l|l|l|l|l|}
\hline $\begin{array}{c}\text { Mois } \\
\text { Anné }\end{array}$ & J & F & M & A & M & J & J & A & S & O & N & D & Total \\
\hline 2015 & - & 3 & 2 & 1 & 1 & 0 & 0 & 1 & 0 & 0 & 1 & 2 & $\mathbf{1 1}$ \\
\hline 2016 & 0 & 0 & 2 & 1 & 1 & 1 & 0 & 0 & 0 & 0 & 2 & 2 & $\mathbf{9}$ \\
\hline 2017 & 0 & 0 & 0 & 2 & 1 & 1 & 2 & 2 & 1 & 0 & 0 & 3 & $\mathbf{1 2}$ \\
\hline Total & 0 & 3 & 4 & 4 & 3 & 2 & 2 & 3 & 1 & 0 & 3 & 7 & 32 \\
\hline
\end{tabular}

Source : Nos enquêtes, 2017

Les mois secs (février à mai ; novembre et décembre), c'est-à-dire lorsque les herbes deviennent rares aux abords du fleuve, sont les périodes où on enregistre le plus de dégâts. En retour, il arrive que des bœufs soient tués ${ }^{5}$ par les paysans. Les conflits sont d'autant plus répétitifs et de plus en plus violents que les règlements nécessitent aussi bien l'intervention des autorités coutumières qu'administratives.

\section{3- La gestion foncière mise à rude épreuve par la récurrence des conflits}

Devant ces faits de conflits qui sont porteurs de germes d'instabilité sociale dans la zone, la gestion foncière est sollicitée en mobilisant les modes de règlement existants.

\section{1- Les différents modes de règlement des conflits}

Pour atténuer les conflits fonciers qui sévissent dans la sous-préfecture d'Ayaou-Sran, les autorités coutumières en collaboration avec les autorités administratives ont mis en place un comité villageois chargé de régler ou de prévenir les conflits. Mais, lorsqu'un cas survient, les protagonistes se rendent d'abord chez les autorités coutumières.

\subsection{1- Le mode de règlement traditionnel}

D'ordinaire, le règlement des conflits fonciers implique les chefs de village et le chef du canton d'Ayaou-Sokpa.

Les chefs de village sont les autorités coutumières chargées de représenter la communauté villageoise et de veiller à la bonne marche des villages. Ils ont en charge de régler notamment les conflits autour de la terre. Selon les chefs de villages interrogés, leurs stratégies se résument d'abord en l'écoute des différents belligérants. Ensuite, il est fait appel à des témoins qui éclairent la prise de décision finale ; c'est-à-dire dans la détermination des acteurs qui ont raison ou non. Mais, il arrive que certains plaignants ne soient pas satisfaits du jugement rendu. Dans ce cas, ils recourent à d'autres juridictions. Il apparaît aussi des situations où le chef de village recommande lui-même aux protagonistes de se rendre à la sous-préfecture après un échec

${ }^{5}$ Le bilan de l'année 2017 fait état de sept bœufs qui ont été tués, selon le chef des éleveurs. 
de résolution (Coulibaly, 2006 : 17). C'est ainsi que de plus en plus, les chefs sont contournés au profit d'autres autorités. Dans les villages d'Ayaou-Sopka, ils ne sont désormais sollicités que pour $24 \%$ des cas de conflits. Selon l'avis des enquêtés, le chef central, la plus grande autorité locale, est quant à lui sollicité (plus de 36\%), pour des cas de tensions répétitives et qui ne trouvent pas d'issues devant les chefs des villages.

Lorsqu'il est sollicité, le chef central entre en contact avec le comité villageois chargé de régler les conflits. Ce comité a pour rôle de déterminer avec des témoins, les limites de la parcelle litigieuse. Il en est de même pour les cas de dégâts causés par les bœufs. C'est à la fin de cette longue procédure que le chef central arrive à trancher l'affaire. Mais, comme le chef de village, le règlement du chef central fait parfois des insatisfaits. Lorsqu'il n'y a pas de compromis entre les protagonistes, l'affaire est conduite chez les autorités administratives, qui sont désormais de plus en plus sollicitées.

\subsection{2- De la faiblesse des autorités coutumières au recours progressif au mode de règlement moderne}

Le règlement moderne des conflits fonciers se fait à la gendarmerie ou à la sous-préfecture. C'est dans de rares cas que l'affaire est portée à la connaissance des autorités judiciaires (Kra, 2015). La gendarmerie est sollicitée dans $12 \%$ des conflits. Selon les paysans, le recours à la gendarmerie est lié à la lenteur constatée dans le règlement traditionnel. Quant au recours au sous-préfet, il est justifié par le fait que les chefs traditionnels sont en perte d'autorité dans les campagnes, comme nous le dit celui d'Akoviébo :

"Avant, le chef de village était bien respecté de tous. Aujourd'hui, qui nous respecte encore ? Maintenant, quand on règle un problème, celui qui a raison dit que le jugement est bon. Et celui qui a tort exprime aussitôt son insatisfaction. C'est ainsi qu'il va solliciter le règlement chez le sous-préfet et ainsi de suite. C'est alors qu'on n'arrive plus à régler les problèmes dans ce village!»

L'une des caractéristiques dans le règlement des conflits, est la remise en cause de la compétence des chefs de village. Il est reproché à ces derniers d'être complices de certaines personnes avec qui, ils entretiennent des rapports assez étroits. Ce propos d'acteur, dans le village de Sadji, en est une illustration :

«Les chefs d'aujourd'hui n'inspirent plus confiance. Ils règlent les problèmes selon leurs rapports qu'ils entretiennent avec chacun des protagonistes. Et puis, ils sont devenus tous jeunes. Et la sagesse manque à tout le monde ! 
Sur la question de limite par exemple, personne ne peut dire clairement qui a raison et qui a tort. Quand c'est comme ça, on ne peut pas s'entendre. »

Si les problèmes fonciers se réglaient autrefois à l'amiable devant les autorités coutumières, la sollicitation $\mathrm{du}$ sous-préfet a pris ainsi de l'importance aujourd'hui. Ainsi, $28 \%$ des enquêtés préfèrent faire appel à cette autorité pour le règlement des conflits afin d'éviter la dégradation des rapports sociaux entre eux.

\section{2- Détérioration des relations entre les communautés villageoises}

Lorsqu'un litige survient entre deux protagonistes d'un même village ou d'une même famille, ces personnes vivent dans une situation de méfiance. Cela se justifie par le fait que les tensions s'expriment soit en la tenue de propos injurieux, soit à la destruction de cultures (arrachage de plants) ou d'autres dégâts. A cela, s'ajoutent les manœuvres autour du mode de règlement. Ils sont nombreux, les paysans qui tolèrent moins le fait d'être traduits devant une juridiction qu'elle soit traditionnelle ou moderne. Ainsi, l'on assiste à une dégradation des relations fraternelles ou de bon voisinage qui liaient ces communautés qui se regardent désormais en chiens de faïence. La cohésion sociale est alors mise à mal entre les habitants des différentes communautés villageoises. De même, les relations entre les agriculteurs ayaous et les éleveurs peuls sont rendues difficiles par l'absence de solution durable à la crise foncière. Dans tous les cas, la cohabitation est dorénavant marquée par la méfiance mutuelle et la rancœur qui augurent de la poursuite de cette tendance conflictuelle dans la zone.

\section{Conclusion}

$\mathrm{Au}$ lendemain de son accession à l'indépendance, l'Etat de Côte d'Ivoire s'est engagé dans une politique hardie d'aménagement de son territoire pour réduire les inégalités socio-économiques entre les régions et améliorer les conditions et le cadre de vie de ses populations. C'est dans cette dynamique que le projet AVB a vu le jour au début des années 1970. Les populations Ayaou installées aux abords du lac ont été sommées de quitter les lieux pour être relocalisées sur de nouveaux sites. Elles ont bénéficié à l'occasion de nombreuses infrastructures sociales et d'un habitat de meilleure qualité. Cependant, près de cinq décennies après leur relocalisation, ces populations font aujourd'hui face à de nombreuses contraintes foncières qui sont sources de nombreux conflits qui engagent une diversité d'acteurs. La mobilisation des différentes autorités traditionnelles comme modernes ne parvient toujours pas à faire baisser la tension, mettant du coup à mal la cohésion sociale entre les communautés. Cette tendance émergente qui, si elle 
se poursuivait, risque de porter un coup à la paix sociale dans toute la zone voire la région mérite une attention particulière de la part des autorités nationales chargées de la question foncière. Ces conflits environnementaux liés à la faible prise en compte de la question foncière dans une perspective de long terme entachent aujourd'hui la durabilité de ce vaste projet d'aménagement du territoire. Dans un tel contexte, le projet AVB se présente comme un cas d'école dont le bilan qui s'impose permettra d'en tirer des leçons utiles aux exercices actuels et à venir d'aménagement du territoire.

\section{References:}

1. Bernardet, Philippe, « Élevage et agriculture dans les savanes du nord. Les mécanismes sociaux d'un conflit», in Eleveurs/Agriculteurs, 1986, pp. 29-40.

2. Boni, Sosthène, «Comprendre l'esprit de la loi $n^{\circ} 98-750$ du 23 Décembre portant code foncier rural en Côte d'Ivoire », in Archives ouvertes, 2015, <hal-01116550>.

3. Coulibaly, Amara, « Gestion des conflits fonciers dans le nord ivoirien Droits, autorités et procédures de règlement des conflits », in Colloque international «Les frontières de la question foncière - At the frontier of land issues », Montpellier, 2006, 19 p.

4. Diop, Mame Dagou, Diédhiou, Cheikh Mamina et Niasse, Madiodio, « Expérience de l'Afrique de l'Ouest en matière de gestion des personnes déplacées par les grands barrages », in Partage des bénéfices issus des grands barrages en Afrique de l'Ouest, Sous la direction de Skinner Jamie, Niasse Madiodio et Haas Lawrence, Série Ressources Naturelles, numéro 19, 2009, 78 p.

5. Gaouli Bi, Anicet Patrice, «Tutorat et Conflits Fonciers Ruraux dans L'ouest Ivoirien : le Cas de Fengolo dans la Sous-préfecture de Duekoué », Consortium for Development Partnerships (CDP), Research report $\mathrm{n}^{\circ}$ 5, 2008, $46 \mathrm{p}$.

6. Hauhouot Asseypo, Antoine, «Développement, Aménagement, Régionalisation en Côte d'Ivoire », Abidjan, EDUCI, 2002, 364p.

7. Homer-Dixon, Thomas, Environment, scarcity and violence, Princeton (NJ): Princeton University Press, 1999, 253 p.

8. Ibo, Jonas, «Phénomène d'acquisition massive des terres et dynamiques socio-foncières en milieu rural ivoirien : enjeux socioéconomiques et culturels ", conférence débat sur les acquisitions massives des terres agricoles en Afrique et les droits des communautés rurales, organisée par Inades-Formation International, 2012, 14 p.

9. INADES-Formation, Les entraves à la mise en xuvre de la loi de 1998 relative au foncier rural en Côte d'Ivoire. Analyse et réflexions 
prospectives, $\quad$ Abidjan, $\quad 2015, \quad 49 \quad$ p. www.inadesfo.net/IMG/pdf/Publication-foncier_rural_complet.pdf.

10. Koffié-Bikpo, Céline Yolande et Adayé, Akoua Assunta, «Géographie des conflits fonciers dans la région du Bas-Sassandra, en Côte d'Ivoire », Revue de la Géographie de l'Université de Ouagadougou, numéro 04, septembre 2015, volume 1, 21 p.

11. Kohlhagen, Dominik, Gestion foncière et conflits entre agriculteurs et éleveurs, autochtones et étrangers dans la région de Korhogo (Côte d'Ivoire), Rapport de mission dans le cadre du projet de recherche « Loi et Coutume » (APREFA - LAJP - CIRAD), 2002, 28 p.

12. Kra, Koffi Simplice, « Le phénomène du « DI-TCHE » et tension dans les zones forestières de la Côte d'Ivoire : Le cas de la sous-préfecture de Ouragahio », Revue de Géographie Tropicale et d'Environnement, n02, 2015, pp. 40-46 (en ligne), consulté le 14 Avril 2017.

13. Lacour, Claude ; Delamarre, Aliette et Thouin, Murielle, 40 ans d'aménagement du territoire, Documentation Française, Paris, 2005, $153 \mathrm{p}$.

14. Lajugie, Joseph ; Delfaud, Pierre et Lacour, Claude, Espace régional et aménagement du territoire, Paris, Dalloz, 1979, 884p.

15. Lassailly-Jacob, Véronique, «Les politiques de transferts de populations liées aux aménagements hydrauliques », Étude comparée de cinq barrages-réservoirs africains, L'Espace géographique, 1983, pp. 46-57.

16. Le Ster, Marine, «Les liens entre conflits et environnement », Les Cahiers d'Outre-Mer [En ligne], 255 | Juillet-Septembre 2011, mis en ligne le 01 juillet 2014, consulté le 14 mai 2017. URL : http://com.revues.org/6339.

17. MINISTERE D'ETAT MINISTERE DU PLAN ET DU DEVELOPPEMENT (MEMPD), 2006, Pré bilan Aménagement du Territoire, Etude réalisée par la DGDER avec l'appui financier et technique de l'UE (PSDAT) août 2006, 91 p.

18. Ori, Boizo, Les villages A.V.B. de l'Ayaou Sud données sociodémographiques, Etudes sur le développement ivoirien, «Société, Développement, Urbanisation », Document de travail, numéro 5, ORSTOM, 1984, 177 p.

19. Oura, Kouadio Raphaël, « Le discours de propagande électorale dans les aires géographiques rurales ivoiriennes : enjeux et pratiques », Nanourougo COULIBALY (éd.), Edition Université de Craiova, 2013, pp. 78-93.

20. PSDU, «Politique en matière de déplacement involontaire de populations », Banque Africaine de développement, Fonds Africain de développement, 2003, 25 p. 
21. Rasoloarison, Ony ; Rakotovao, Jean-Marie et Bockel, Louis, Accès au capital, crédit, accès au foncier et pauvreté rurale à Madagascar, Note d'analyse UPDR, Version 3 jeudi 9 août 2001, ged.ubordeaux4.fr/SDROSOLOARISON.pdf 